\title{
Testing exponentiality against renewal new better than used in Laplace transform order: applications in reliability
}

\author{
M. A. W. Mahmoud', L. S. Diab² and D. M. Radi ${ }^{3^{*}}$
}

* Correspondence: doaa.m.radi@
mhiet.edu.eg
${ }^{3}$ Department of Basic Science
(Mathematics), El Minya High
Institute for Engineering and
Technology, El-Minia 61111, Egypt
Full list of author information is
available at the end of the article

\begin{abstract}
In this paper, a new class of life distribution is proposed, named renewal new better than used in Laplace transform order (RNBUL). The test statistic for testing exponentiality versus (RNBUL) based on U-statistic is proposed. Pitman's asymptotic efficiencies of the test and Pitman's asymptotic relative efficiencies (PARE) are calculated. The percentiles of this test statistic are tabulated, and the powers of this test are estimated for some famous alternatives distributions in reliability such as Weibull, linear failure rate (LFR), and Gamma distributions. The problem regarding the right-censored data is also handled. Finally, some applications to elucidate the usefulness of the proposed test in reliability analysis are discussed.
\end{abstract}

Keywords: Classes of life distributions, RNBUL, Testing Exponentiality, U test, Pitman asymptotic efficiency, Censored data

AMS 2010 Subject Classification: 62N05, 62E99, 62G10

\section{Introduction and motivation}

It is known that the theory of reliability is a branch of statistical technology that deals with general regularities. The importance of reliability appears in its dealing with the length of human beings, organisms' structures, materials, etc. This theory is widely used in biological, engineering and medicine sciences. The reliability of effective origins has been considered by the works that participated in the reliability theory by $[1,2]$. The statisticians and reliability analysts have used classifications of life distributionsbased on some aspects of aging-to model survival data. The aging concepts function is to describe how a population of units or systems improves or deteriorates with age.

Aging properties is the means to define the life distribution classes. The exponential distribution is one of the most important aspects of such classifications, and it is always a member of each class. The notion of aging has great importance in many reliability analyses. On the other hand, many statistics have been developed for testing exponentiality against different aging alternatives.

The main classes of life distributional are based on new better than used NBU, new better than used failure rate NBUFR, new better than average failure rate NBAFR, new better than used renewal failure rate NBURFR, new better than renewal used NBRU, and exponentially better than used in Laplace transform order EBUL. Many

(c) The Author(s). 2019 Open Access This article is distributed under the terms of the Creative Commons Attribution 4.0 International License (http://creativecommons.org/licenses/by/4.0/), which permits unrestricted use, distribution, and reproduction in any medium, provided you give appropriate credit to the original author(s) and the source, provide a link to the Creative Commons license, and indicate if changes were made. 
researchers have introduced testing exponentiality against some classes of life distributions. For testing exponentiality versus NBU class, see [3]. A new class of life distributions named NBUCL has introduced by [4]. The classes NBAFR, NBARFR, NBURFR, and RNBRUE have proposed by [5-8], respectively.

\section{Renewal classes}

Consider a device (system or component) with lifetime $T$ and continuous life distribution $F(t)$, is put on the operation. When the failure occurs, the device will be replaced by a sequence of mutually independent devices. The spare devices are independent of the first device and identically distributed with the same life distributions $F(t)$. In the long run, the remaining life distribution of the system under operation at time $t$ is given by stationary renewal distribution as follows:

$$
W_{F}(t)=\mu_{F}^{-1} \int_{0}^{t} \bar{F}(u) d u, 0 \leq t<\infty,
$$

where $\mu_{F}=\mu=\int_{0}^{\infty} \bar{F}(u) d u$. The corresponding renewal survival function is

$$
\bar{W}_{F}(t)=\mu_{F}^{-1} \int_{t}^{\infty} \bar{F}(u) d u, 0 \leq t<\infty,
$$

For details, see $[5,9]$.

The NRBU, RNBU, NRBUE, and HNRBUE classes of life distributions have introduced by [10], and the relation between them has been studied. Testing exponentiality versus NRBU based on TTT-transform has been investigated by [11]. A new test statistic for testing exponentiality against RNBU class of life distribution based on $U$-statistic is studied by [12].

\section{Definition 1}

If $\mathrm{X}$ is a random variable with survival function $\bar{F}(x)$, then $\mathrm{X}$ is said to have renewal new better (worth) than used property, denoted by RNBU (RNWU), if

$$
\bar{W}_{F}(x+t) \leq(\geq) \bar{W}_{F}(x) \bar{W}_{F}(t) x \geq 0, t \geq 0 .
$$

Now, a new class of life distributions called renewal new better than used in Laplace transform order has been defined by [13].

\section{Definition 2}

$X$ is said to be renewal new better than used in Laplace transform order (RNBUL) if:

$$
\int_{0}^{\infty} e^{-s x} \bar{W}_{F}(x+t) d x \leq \bar{W}_{F}(t) \int_{0}^{\infty} e^{-s x} \bar{W}_{F}(t) d x, x \geq 0, t \geq 0 .
$$

Testing exponentiality against RNBUL

In this section, we test the null hypotheses $H_{0}: F$ is exponential with mean $\mu$ against $H_{1}: F$ belongs to RNBUL and not exponential.

The following lemma is essential for the development of our test statistic. 
Lemma 2.1.

Let $X$ be $R N B U L$ random variable with distribution function $F$, then

$$
\frac{\mu}{s^{3}}-\frac{\mu}{s^{3}} E\left(e^{-s X}\right)-\frac{\mu^{2}}{s^{2}} \leq \frac{\mu_{2}}{2 s^{2}} E\left(e^{-s X}\right)-\frac{\mu_{2}}{2 s^{2}},
$$

where

$$
E\left(e^{-s X}\right)=\int_{0}^{\infty} e^{-s x} d F(x)
$$

Proof.

Since $F$ belongs to RNBUL, then from Definition 2 and Integrating both sides with respect to $t$ over $[0, \infty)$, gives

$$
\int_{0}^{\infty} \int_{0}^{\infty} e^{-s x} \bar{W}_{F}(x+t) d x d t \leq \int_{0}^{\infty} \bar{W}_{F}(t) \int_{0}^{\infty} e^{-s x} \bar{W}_{F}(t) d x d t
$$

setting

$$
I=\int_{0}^{\infty} \int_{0}^{\infty} e^{-s x} \bar{W}_{F}(x+t) d x d t
$$

Therefore,

$$
I=\frac{1}{\mu} E \int_{0}^{X}\left[\frac{1}{s} X+\frac{1}{s^{2}} e^{-s(X-t)}-\frac{1}{s^{2}}-\frac{1}{s} t\right] d t,
$$

Then,

$$
I=\frac{\mu_{2}}{2 s \mu}-\frac{1}{s^{3} \mu} E\left(e^{-s X}\right)-\frac{1}{s^{2}}+\frac{1}{s^{3} \mu} .
$$

Similarly,

$$
I I=\int_{0}^{\infty} \bar{W}_{F}(t) \int_{0}^{\infty} e^{-s x} \bar{W}_{F}(t) d x d t
$$

Then,

$$
I I=\frac{\mu_{2}}{2 s \mu}-\frac{\mu_{2}}{2 s^{2} \mu^{2}}+\frac{\mu_{2}}{2 s^{2} \mu^{2}} E\left(e^{-s X}\right) .
$$

Substituting (2.3) and (2.4) in (2.2), we get (2.1). This completes the proof.

\section{Empirical test statistic for RNBUL}

Let $X_{1}, X_{2}, X_{n}$ be a random sample from a population with a distribution function $F \in$ RNBUL class. The measure of departure from exponentiality $\delta(s)$ is determined from the previous lemma, where

$$
\delta(s)=\frac{\mu_{2}}{2 s^{2}} E\left(e^{-s X}\right)-\frac{\mu_{2}}{2 s^{2}}+\frac{\mu}{s^{3}} E\left(e^{-s X}\right)+\frac{\mu^{2}}{s^{2}}-\frac{\mu}{s^{3}} .
$$


Note that under $H_{0}: \delta(s)=0$, and $H_{1}: \delta(s)>0$.Let $\hat{\delta}(s)$ be the empirical estimate of $\delta(s)$, where

$$
\hat{\delta}(s)=\frac{1}{n(n-1)} \sum_{i=1}^{n} \sum_{j=1}^{n}\left[\frac{X_{i}^{2}}{2 s^{2}} e^{-s X_{j}}-\frac{X_{i}^{2}}{2 s^{2}}+\frac{X_{i}}{s^{3}} e^{-s X_{j}}+\frac{X_{i} X_{j}}{s^{2}}-\frac{X_{i}}{s^{3}}\right],
$$

To make the test scale-invariant under $H_{0}$, we use $\hat{\Delta}(s)=\frac{\hat{\delta}(s)}{\bar{X}^{2}}$, where $\bar{X}=\frac{1}{n} \sum_{i=1}^{n} X_{i}$ is the sample mean. Then,

$$
\hat{\Delta}(s)=\frac{1}{n(n-1) \bar{X}^{2}} \sum_{i=1}^{n} \sum_{j=1}^{n}\left[\frac{X_{i}^{2}}{2 s^{2}} e^{-s X_{j}}-\frac{X_{i}^{2}}{2 s^{2}}+\frac{X_{i}}{s^{3}} e^{-s X_{j}}+\frac{X_{i} X_{j}}{s^{2}}-\frac{X_{i}}{s^{3}}\right] .
$$

Setting

$$
\phi\left(X_{1}, X_{2}\right)=\frac{X_{1}^{2}}{2 s^{2}} e^{-s X_{2}}-\frac{X_{1}^{2}}{2 s^{2}}+\frac{X_{1}}{s^{3}} e^{-s X_{2}}+\frac{X_{1} X_{2}}{s^{2}}-\frac{X_{1}}{s^{3}},
$$

and defining the symmetric kernel

$$
\psi\left(X_{1}, X_{2}\right)=\frac{1}{2 !} \sum \phi\left(X_{1}, X_{2}\right),
$$

where the summation is over all arrangements of $X_{1}, X_{2}, X_{n}$, then $\hat{\delta}(s)$ is equivalent to $U$-statistic

$$
U_{n}=\frac{1}{\left(\frac{n}{2}\right)} \sum \phi\left(X_{1}, X_{2}\right) .
$$

The following theorem summarizes the asymptotic properties of the test.

\section{Theorem 2.1}

(i) As $n \rightarrow \infty, \sqrt{n}[\hat{\Delta}(s)-\delta(s)]$ is asymptotically normal with mean zero and variance

$$
\sigma^{2}(s)=\operatorname{Var}\left\{\left(E\left(e^{-s X}\right)-1\right)\left(\frac{X^{2}}{2 s^{2}}-\frac{X}{s^{3}}\right)+\left(e^{-s X}-1\right)\left(\frac{\mu_{2}}{2 s^{2}}+\frac{\mu}{s^{3}}\right)+\frac{2 X \mu}{s^{2}}\right\} .
$$

(ii) Under $H_{0}$ the variance reduced to

$$
\sigma_{0}^{2}=\frac{2}{(1+s)^{3}(1+2 s)}, s \neq-1,-\frac{1}{2}
$$

Proof:

Using standard $U$-statistics theory, see [14], and by direct calculations, we can find the mean and the variance as follows: 


$$
\sigma^{2}=\operatorname{Var}\{\eta(X)\}
$$

Where $\eta(X)=\eta_{1}(X)+\eta_{2}(X)$,

$$
\begin{aligned}
& \eta_{1}(X)=E\left[\phi\left(X_{1}, X_{2}\right) \mid X_{1}\right] \\
& =\frac{X^{2}}{2 s^{2}} E\left(e^{-s X}\right)-\frac{X^{2}}{2 s^{2}}+\frac{X}{s^{3}} E\left(e^{-s X}\right)+\frac{X \mu}{s^{2}}-\frac{X}{s^{3}}
\end{aligned}
$$

and

$$
\begin{aligned}
& \eta_{2}(X)=E\left[\phi\left(X_{1}, X_{2}\right) \mid X_{2}\right] \\
& =\frac{\mu_{2}}{2 s^{2}} e^{-s X}-\frac{\mu_{2}}{2 s^{2}}+\frac{\mu}{s^{3}} e^{-s X}+\frac{X \mu}{s^{2}}-\frac{\mu}{s^{3}},
\end{aligned}
$$

Therefore,

$$
\eta(X)=\left(E\left(e^{-s X}\right)-1\right)\left(\frac{X^{2}}{2 s^{2}}-\frac{X}{s^{3}}\right)+\left(e^{-s X}-1\right)\left(\frac{\mu_{2}}{2 s^{2}}+\frac{\mu}{s^{3}}\right)+\frac{2 X \mu}{s^{2}}
$$

and Eq. (2.8) is deduced.

Under $H_{0}$, the mean $\mu_{0}$ and the variance $\sigma_{0}^{2}$ are given by

$$
\begin{gathered}
\mu_{0}=E\left(\eta_{0}(X)\right)=0, \\
\sigma_{0}{ }^{2}=E\left[\left(\eta_{0}(X)\right)^{2}\right],
\end{gathered}
$$

Then, (2.9) is obtained.

\section{The Pitman asymptotic efficiency}

To access the quality of the test, Pitman asymptotic efficiencies (PAE's) are computed and compared with some other tests for the following alternative:

\section{i. The Weibull family:}

$$
\bar{F}_{1}(x)=e^{-x^{\theta}}, x \geq 0, \theta \geq 1 \text {. }
$$

\section{ii. The linear failure rate family:}

$$
\bar{F}_{2}(x)=e^{-x-\frac{\theta}{2} x^{2}}, x \geq 0, \theta \geq 0 .
$$

\section{iii. The Makeham family:}

$$
\bar{F}_{3}(x)=e^{\left[-x-\theta\left(x+e^{-x}+1\right)\right]}, x \geq 0, \theta \geq 0 .
$$

Note that for $\theta=1, F_{1}$ goes to exponential distribution and for $\theta=0, F_{2}$ and $F_{3}$ reduce to the exponential distributions. The PAE is defined by

$$
\operatorname{PAE}(\hat{\Delta}(s))=\frac{1}{\sigma_{0}}\left[\frac{d}{d \theta} \delta(s)\right]_{\theta \rightarrow \theta_{0}},
$$

when $s=2$, this leads to

$$
\operatorname{PAE}[\hat{\Delta}(2), \text { Weibull }]=0.618, \operatorname{PAE}[\hat{\Delta}(2), \mathrm{LFR}]=0.915
$$

and $\operatorname{PAE}[\hat{\Delta}(2)$, Makeham $]=0.172$, where $\sigma_{0}(2)=0.121716$. The Pitman asymptotic rela- 
tive efficiency (PARE) of our test $\hat{\Delta}(s)$ comparing to $\delta_{1}, \delta_{2}, \delta_{3}$, and $\delta_{4}$ which were studied by $[6,8,15,16]$, respectively, is calculated where

$$
\operatorname{PARE}\left(T_{1}, T_{2}\right)=\frac{\operatorname{PAE}\left(T_{1}\right)}{\operatorname{PAE}\left(T_{2}\right)}
$$

We can see from Table 1 that our test statistic $\hat{\Delta}(s)$ for RNBUL is more efficient than the other four cases.

\section{Monte Carlo null distribution critical values}

In this section, we have simulated the upper percentile points for 90th, 95th, 98th, and 99th based on 5000 simulated samples of sizes $n=5(5) 50,11$ from the standard exponential distribution by using MATHEMATICA 10.

It can be noticed from Table 2 and Fig. 1 that the critical values are increasing as confidence levels increase and decrease as the sample size increases

\section{Power estimates of the test $\hat{\Delta}(s)$}

The power of the statistic $\hat{\Delta}(s)$ will be carried out using significant level $\alpha=0.05$ with appropriate parameter values of $\theta$ at $n=10,20$, and 30 for 3 frequently used distributions such as Weibull, linear failure rate, and Gamma distributions based on 5000 simulated samples tabulated in Table 3.

From Table 3, we see that our test $\hat{\Delta}(s)$ has very good power for all alternatives.

\section{Testing for censored data}

A test statistic is proposed to test $H_{0}$ versus $H_{1}$ in the case of randomly right-censored (RR-C) data in many practical experiments; the censored data are the only information available in a life-testing model or in a clinical study where patients may be lost (censored) before the completion of a study. This experimental situation can formally be modeled as follows: suppose $n$ units are put on test, and $X_{1}, X_{2}, \ldots, X_{n}$ denote their true-lifetime which are independent, identically distributed (i.i.d.) according to continuous life distribution $F$. Let $Y_{1}, Y_{2}, \ldots, Y_{n}$ be (i.i.d.) according to continuous life distribution G. $X \mathrm{~s}$ and $Y \mathrm{~s}$ are assumed to be independent. In the RR-C model, we observe the pairs $\left(Z_{j}, \delta_{j}\right), j=1,2, \ldots, n$ where $Z_{j}=\min \left(X_{j}, Y_{j}\right)$ and

$$
\delta_{j}=\left\{\begin{array}{c}
1 \text { if } Z_{j}=X_{j}(j \text { th observation is uncensored }) \\
0 \text { if } Z_{j}=Y_{j}(j \text { th observation is censored })
\end{array}\right.
$$

Let $Z_{(0)}=0<Z_{(1)}<Z_{(2)}<\ldots<Z_{(n)}$ denote the ordered $Z$ s and $\delta_{(j)}$ is the $\delta_{j}$ corresponding to $Z_{(j)}$. Using censored data $\left(Z_{j}, \delta_{j}\right), j=1,2, \ldots, n$ [17]. proposed the product-limit estimator,

Table 1 The asymptotic relative efficiencies for our test versus $\delta_{i}, i=1,2,3,4$

\begin{tabular}{llll}
\hline Test & Wiebull & LFR & Makeham \\
\hline PARE $\left(\hat{\Delta}(s), \delta_{1}\right)$ & 4.682 & 2.113 & 1.198 \\
PARE $\left(\hat{\Delta}(s), \delta_{2}\right)$ & 3.653 & 2.243 & 4.419 \\
$\operatorname{PARE}\left(\hat{\Delta}(s), \delta_{3}\right)$ & 2.771 & 1.71 & 0.937 \\
$\operatorname{PARE}\left(\hat{\Delta}(s), \delta_{4}\right)$ & 12.36 & 4.217 & 1.197 \\
\hline
\end{tabular}


Table 2 The upper percentile of $\hat{\Delta}(s)$ with 5000 replications at $s=2$

\begin{tabular}{lllll}
\hline$n$ & $90 \%$ & $95 \%$ & $98 \%$ & $99 \%$ \\
\hline 5 & 0.0394076 & 0.0449232 & 0.0513921 & 0.0567619 \\
10 & 0.0310125 & 0.0348235 & 0.0397779 & 0.0429144 \\
11 & 0.0310125 & 0.0348235 & 0.0397779 & 0.0429144 \\
15 & 0.0272512 & 0.0309445 & 0.0347753 & 0.0375317 \\
20 & 0.0250087 & 0.0282498 & 0.0316245 & 0.0340623 \\
25 & 0.0233536 & 0.0263381 & 0.0293944 & 0.0315939 \\
30 & 0.0215709 & 0.0244302 & 0.0274829 & 0.030076 \\
35 & 0.020248 & 0.0231756 & 0.0259756 & 0.0282651 \\
40 & 0.0193352 & 0.0223912 & 0.0250982 & 0.0267005 \\
45 & 0.0185366 & 0.0209917 & 0.0237138 & 0.0255735 \\
50 & 0.0178576 & 0.0205212 & 0.0232389 & 0.025033 \\
\hline
\end{tabular}

$$
\bar{F}_{n}(X)=\prod_{\left[j: Z_{(j)} \leq X\right]}\{(n-j)(n-j+1)\}^{\delta_{(j)}}, X \in\left[0, Z_{(j)}\right] .
$$

Now, for testing $H_{0}: \delta(s)=0$ against $H_{1}: \delta(s)>0$, using randomly right-censored data, we propose the following test statistic

$$
\hat{\Delta}_{c}(s)=\frac{1}{\mu^{2}}\left[\left(\frac{\mu_{2}}{2 s^{2}}+\frac{\mu}{s^{3}}\right) E\left(e^{-s X}\right)-\frac{\mu_{2}}{2 s^{2}}+\mu\left(\frac{\mu}{s^{2}}-\frac{1}{s^{3}}\right)\right] .
$$

For computational purposes, (6.1) may be rewritten as

$$
\hat{\Delta}_{c}(s)=\frac{1}{\Phi^{2}}\left[\left(\frac{\Omega}{2 s^{2}}+\frac{\Phi}{s^{3}}\right) \Theta-\frac{\Omega}{2 s^{2}}+\Phi\left(\frac{\Phi}{s^{2}}-\frac{1}{s^{3}}\right)\right],
$$

where

$$
\begin{aligned}
& \Phi=\sum_{k=1}^{n}\left[\prod_{m=1}^{k-1} C_{m}^{\delta(m)}\left(Z_{(k)}-Z_{(k-1)}\right)\right], \\
& \Omega=2 \sum_{i=1}^{n}\left[\prod_{v=1}^{i-1} Z_{(i)} C_{v}^{\delta(v)}\left(Z_{(i)}-Z_{(i-1)}\right)\right],
\end{aligned}
$$

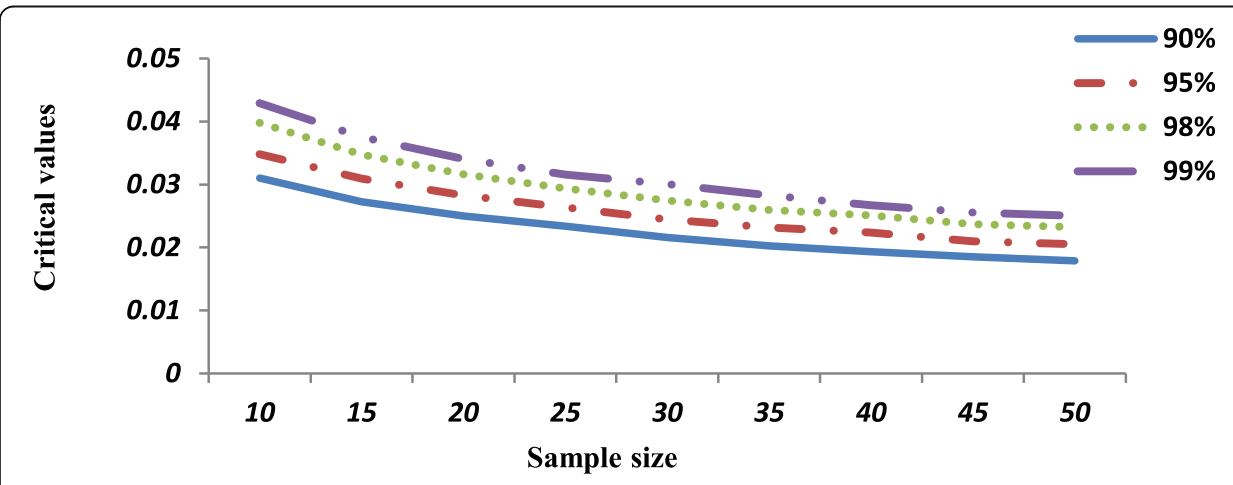

Fig 1 Relation between critical values, sample size and confidence levels 
Table 3. Power estimates of the statistic $\hat{\Delta}(s)$

\begin{tabular}{|c|c|c|c|c|}
\hline \multirow[t]{2}{*}{ Distribution } & \multirow{2}{*}{$\begin{array}{l}\text { Parameter } \\
\theta\end{array}$} & \multicolumn{3}{|c|}{ Sample size } \\
\hline & & $n=10$ & $n=20$ & $n=30$ \\
\hline \multirow[t]{3}{*}{ LFR } & 2 & 1.0000 & 1.0000 & 1.0000 \\
\hline & 3 & 1.0000 & 1.0000 & 1.0000 \\
\hline & 4 & 1.0000 & 1.0000 & 1.0000 \\
\hline \multirow[t]{3}{*}{ Weibull } & 2 & 1.0000 & 1.0000 & 1.0000 \\
\hline & 3 & 1.0000 & 1.0000 & 1.0000 \\
\hline & 4 & 1.0000 & 1.0000 & 1.0000 \\
\hline \multirow[t]{3}{*}{ Gamma } & 2 & 0.9858 & 0.9938 & 0.9999 \\
\hline & 3 & 0.9986 & 0.9998 & 1.0000 \\
\hline & 4 & 0.9998 & 1.0000 & 1.0000 \\
\hline
\end{tabular}

and

$$
d x=\left(Z_{(j)}-Z_{(j-1)}\right), C_{k}=[n-k][n-k+1]^{-1} .
$$

Table 4 below gives the critical values percentiles of $\hat{\Delta}_{c}(s)$ test for sample size $n=$ 5(5)30(10), 81, 86.

It can be noticed from Table 4 and Fig. 2 that the critical values are increasing as confidence levels increase and decrease as the sample size increases

\section{Power estimates of the test $\hat{\Delta}_{c}(s)$}

The power of the statistic $\hat{\Delta}_{c}(s)$ is considered at the significant level $\alpha=0.05$ with suitable parameter values of $\theta$ at $n=10,20$, and 30 for some of the most commonly used distributions such as Weibull and linear failure rate distributions based on 5000 simulated samples tabulated in Table 5.

From Table 5, we see that our test $\hat{\Delta}_{c}(s)$ has good power for all alternatives.

Table 4. The upper percentile of $\hat{\Delta}_{c}(s)$ with 5000 replications at $s=2$

\begin{tabular}{lllll}
\hline$n$ & $90 \%$ & $95 \%$ & $98 \%$ & $99 \%$ \\
\hline 5 & 0.0476514 & 0.0575673 & 0.0686764 & 0.0780609 \\
10 & 0.0286204 & 0.0415836 & 0.0552211 & 0.0655845 \\
15 & 0.023596 & 0.0349254 & 0.046839 & 0.0555851 \\
20 & 0.0190485 & 0.0300757 & 0.0419818 & 0.0477634 \\
25 & 0.018172 & 0.0277966 & 0.0388292 & 0.0445861 \\
30 & 0.0175909 & 0.0268114 & 0.0363807 & 0.0421265 \\
40 & 0.0143751 & 0.0225325 & 0.0319051 & 0.037132 \\
50 & 0.0129596 & 0.0208445 & 0.0293955 & 0.0348187 \\
60 & 0.0112546 & 0.0188828 & 0.0274677 & 0.0314528 \\
70 & 0.0107728 & 0.016817 & 0.0235266 & 0.029162 \\
81 & 0.00952946 & 0.0150434 & 0.0217278 & 0.0249294 \\
86 & 0.00860122 & 0.0142955 & 0.0206852 & 0.0246744 \\
\hline
\end{tabular}




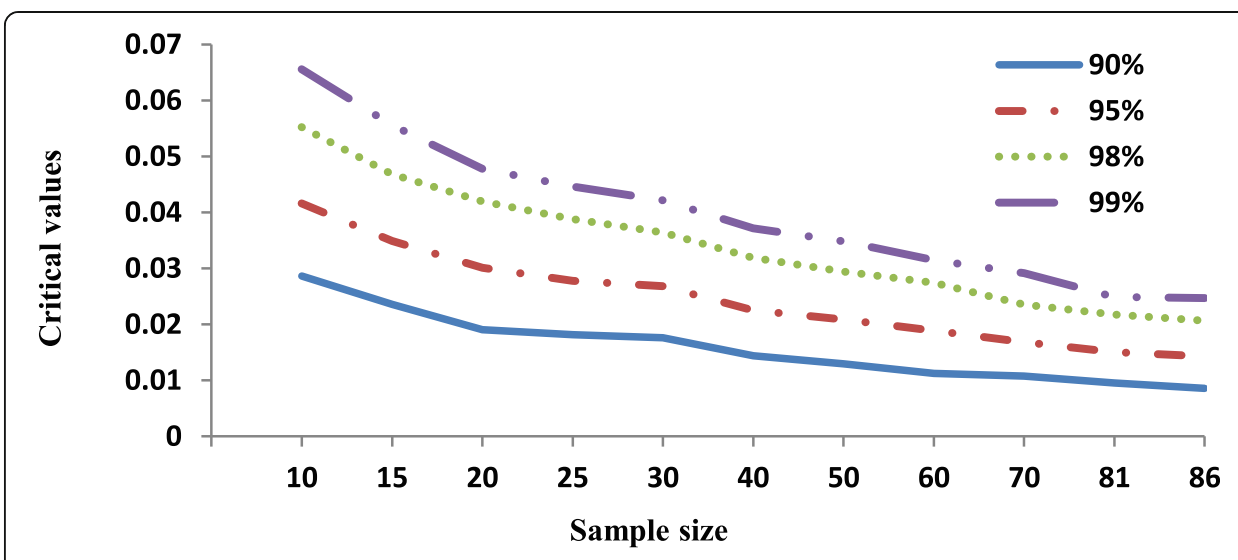

Fig 2 Relation between critical values, sample size and confidence levels

\section{Some applications}

In this section, we apply the test on some datasets to elucidate the applications of the RNBUL in both non-censored data and censored data at 95\% confidence level.

\section{Complete data}

\section{Dataset \#1}

Consider the dataset given in [18] and have been used in [19]. This dataset gives the times between arrivals of 25 customers at a facility. In this case, we get $\hat{\Delta}(s)=0.08892$ 4 greater than the critical value of Table 2 . Hence, we reject the null hypothesis $H_{0}$ and accept $H_{1}$ which states that the data have RNBUL property.

\section{Dataset \#2}

Consider the data-set given in [20] which represents failure times in hours, for a specific type of electrical insulation in an experiment in which the insulation was subjected to a continuously increasing voltage stress, then we get $\hat{\Delta}(s)=0.0242465$ which is less than the critical value of Table 2. Hence, we accept the null hypothesis $H_{0}$.

\section{Censored data}

Dataset \#3

Consider the dataset in [21]. These data represent 81 survival times of patients of melanoma. Out of these, 46 represents the whole times (non-censored data). We get $\hat{\Delta}_{c}(s)=-1.1$

Table 5 Power estimates of the statistic $\hat{\Delta}_{c}(s)$

\begin{tabular}{llllr}
\hline Distribution & Parameter $\theta$ & \multicolumn{2}{l}{ Sample size } & \\
\cline { 3 - 5 } & & $n=10$ & $n=20$ & $n=30$ \\
\hline LFR & 2 & 1.0000 & 1.0000 & 1.0000 \\
& 3 & 1.0000 & 1.0000 & 1.0000 \\
& 4 & 1.0000 & 1.0000 & 1.0000 \\
Weibull & 2 & 0.9992 & 0.9998 & 1.0000 \\
& 3 & 1.0000 & 1.0000 & 1.0000 \\
& 4 & 1.0000 & 1.0000 & 1.0000 \\
\hline
\end{tabular}


$7837 \times 10^{87}$ which is less than the critical value of Table 4 at $95 \%$ upper percentile. Hence, we accept the null hypothesis $H_{0}$.

\section{Dataset \#4}

Consider the dataset given in [22] for lung cancer patients. These data consist of 86 survival times (in months) with 22 right-censored. In this case, we get $\hat{\Delta}_{c}(s)=-4.1890$ $7 \times 10^{85}$ which is less than the critical value of Table 4 at $95 \%$ upper percentile. Hence, we accept the null hypothesis $H_{0}$.

\section{Abbreviations}

EBUL: Exponential better than used in Laplace transform order; LFR: Linear failure rate; NBAFR: New better than average failure rate; NBARFR: New better than average renewal failure rate; NBRU: New better than renewal used; NBU: New better than used; NBUCL: New better than used in increasing convex in Laplace transform order; NBURFR: New better than used renewal failure rate; PARE: Pitman's asymptotic relative efficiencies; RNBRUE: Renewal new better than renewal used in expectation; RNBUL: Renewal new better than used in Laplace transform order

\section{Authors' contributions}

All authors contributed equally to this manuscript. All authors read and approved the final manuscript.

\section{Funding}

The authors received no financial support for the research, authorship, and/or publication of this article.

\section{Availability of data and materials}

All the data in the manuscript are public.

\section{Competing interests}

The authors declare that they have no competing interests.

\section{Author details}

${ }^{1}$ Department of Mathematics, Faculty of Science, Al-Azhar University, Nasr City 11884, Egypt. ${ }^{2}$ Department of Mathematics, Faculty of Science, Al-Azhar University (Girls branch), Nasr City 11884, Egypt. ${ }^{3}$ Department of Basic Science (Mathematics), El Minya High Institute for Engineering and Technology, El-Minia 61111, Egypt.

Received: 1 April 2019 Accepted: 27 September 2019

Published online: 04 December 2019

\section{References}

1. Barlow, R.E., Marshall, A.W., Proschan, F.: Properties of probability distributions with monotone hazard rate. Ann Math Stat. 34(2), 375-389 (1963)

2. Bryson, M.C., Siddiqui, M.: Some criteria for aging. J Am Stat Assoc. 64(328), 1472-1483 (1969)

3. Ahmed, l.: A class of statistics useful in testing increasing failure rate average and new beter than used life distribution. $J$ Statist Plant Inf. 41, 141-149 (1994)

4. Mahmoud, M.A.W., Diab, L. S and Radi, D. M, A Nonparametric Test for Testing NBUCL Class of life distributions with Applications. IOSR J Math, 2019. 15(1): p. 15-21.

5. Abouammoh, A., Ahmed, A.: On renewal failure rate classes of life distributions. Stat Prob Lett. 14(3), $211-217$ (1992)

6. Mahmoud, M.A.W., Alim, N.A.A.: On testing exponentiality against NBARFR life distribution. Statistica. 62(4), 619-631 (2007)

7. Mahmoud, M., Alim, N.A.: A goodness of fit approach for Testing NBUFR (NWUFR) and NBAFR (NWAFR) properties. Int J Rel Appl. 9(2), 125-140 (2008)

8. Abdel Aziz, A.: On testing exponentiality against RNBRUE alternatives. Appl Math Sci. 35, 1725-1736 (2007)

9. Barlow, R. and F. Proschan, Statistical theory of Reliability and life testing. To begin with. Silver Spring, MD, 1981.

10. Abouammoh, A., Ahmad, R., Khalique, A.: On new renewal better than used classes of life distributions. Stat Prob Lett. 48(2), 189-194 (2000)

11. Abouammoh, A. and A. Khalique, On tests of new renewal better than used classes of life distributions. Parisan. Samikkha, 1998. 5: p. 21-32.

12. Mahmoud, M., El-Arishy, S., Diab, L.: Testing renewal new better than used life distributions based on U-test. Appl Math Model. 29(8), 784-796 (2005)

13. M. A. W. Mahmoud, L.S.D.a.D.M.R., Testing Exponentiality Against RNBUL Class of Life Distribution Based on Goodness of Fit. J. Stat. Appl. Pro., 2019. 8(1): p. 57-66.

14. Lee, A.J.: U-statistics: Theory and Practice. Marcel Dekker, New York (1990)

15. Kango, A.: Testing for new is better than used. Comm Statist Theory Methods. 12, 311-321 (1993)

16. Mugdadi, A., Ahmad, I.A.: Moment inequalities derived from comparing life with its equilibrium form. I Stat Plan Inference. 134(2), 303-317 (2005)

17. Kaplan, E.L., Meier, P.: Nonparametric estimation from incomplete observations. J Am Stat Assoc. 53(282), 457-481 (1958)

18. Grubbs, F.E.: Approximate fiducial bounds on reliability for the two parameter negative exponential distribution. Technometrics. 13(4), 873-876 (1971)

19. Shapiro, S., Goodness of Fit Tests in the Exponential Distribution Theory Methods and Applications. Balakrishnan, N.; Basu, AP Editors. 1995, Gordon and Breach, Amsterdam. 
20. Lawless, J.F., Statistical models and methods for lifetime data. Vol. 362. 2011: John Wiley \& Sons.

21. Susarla, V. and J. Van Ryzin, Empirical Bayes estimation of a distribution (survival) function from right censored observations. Ann Stat, 1978: p. 740-754.

22. Pena, A.: Goodness of fit tests with censored data (2002)

\section{Publisher's Note}

Springer Nature remains neutral with regard to jurisdictional claims in published maps and institutional affiliations.

Submit your manuscript to a SpringerOpen ${ }^{\odot}$ journal and benefit from:

- Convenient online submission

- Rigorous peer review

- Open access: articles freely available online

- High visibility within the field

- Retaining the copyright to your article

Submit your next manuscript at $\boldsymbol{\nabla}$ springeropen.com 